\title{
PARTICLE INTERACTIONS IN THERMOPHORESIS
}

\author{
HUAN J. $\mathrm{KEH}^{\dagger}$ and SHIH H. CHEN \\ Department of Chemical Engineering, National Taiwan University, Taipei 106-17, Taiwan, Republic of \\ China
}

(First received 23 March 1995; revised manuscript received and accepted 22 May 1995)

\begin{abstract}
An analytical study is presented for the thermophoretic motion of two freely suspended aerosol spheres using a method of reflections. The particles are oriented arbitrarily with respect to the prescribed constant temperature gradient, and they are allowed to differ in radius, in thermal conductivity and in surface properties. The Knudsen numbers are assumed to be small so that the fluid flow is described by a continuum model with a thermal creep and a hydrodynamic slip at the particle surfaces. The method of refiections is based on an analysis of the thermal and hydrodynamic disturbances produced by a single sphere placed in an arbitrarily varying temperature field. The results for two-sphere interactions are correct to $O\left(r_{12}^{-7}\right)$, where $r_{12}$ is the distance between the particle centers. For the special case of spheres oriented along the undisturbed temperature gradient, our results are in perfect agreement with the numerical solution obtained using bipolar coordinates. Based on a microscopic model the results for two-sphere interactions are used to find the effect of the volume fraction of particles of each type on the average thermophoretic velocity in a bounded suspension. For a suspension of identical spheres, this average velocity is reduced as the particle concentration is increased. In general, the effect of particle interactions on thermophoresis is much weaker than that on sedimentation.
\end{abstract}

\section{INTRODUCTION}

Small particles suspended in a gas in which there is a temperature gradient acquire a mean velocity relative to the gas in the direction of decreasing temperature. This phenomenon, known as thermophoresis of aerosol particles, was first described by Tyndall in 1870 (Waldmann and Schmitt, 1966; Bakanov, 1991). He observed a dust-free zone in a dusty gas around a hot body; this zone could be seen as a dark space if properly illuminated. A common example of thermophoresis is the blackening of the glass globe of a kerosene lantern; the temperature gradient established between the flame and the globe drives the carbon particles produced in the combustion process toward the globe, where they deposit.

Deposition by thermophoresis is of considerable practical importance in many industrial applications when hot gases containing small suspended particles flow over cool surfaces. For example, thermophoresis can be effective in removing or collecting small particles from laminar gas streams in air cleaning and aerosol sampling devices (Batchelor and Shen, 1985; Sasse et al., 1994). The phenomenon has also been cited as an origin for the deposition of particulate matter on surfaces of heat exchangers causing scale formation with the attendant reduction of the heattransfer coefficient (Friedlander, 1977; Montassier et al., 1991). Convincing evidence has been provided that, in the modified chemical vapor deposition process for the manufacture of high-quality optical fibers, thermophoresis is the primary mechanism responsible

${ }^{\dagger}$ Corresponding author. for the deposition of aerosol particles (soot) onto the inner walls of the containing tube (Simpkins et al., 1979; Weinberg, 1982). On the other hand, deposition of contaminant particles by thermophoresis on wafers in clean rooms during manufacturing steps can be a major cause of loss of product yields in the microelectronics industry (Ye et al., 1991). In the area of nuclear safety; knowledge of thermophoresis is required to calculate the deposition rates of radioactive aerosol particles released in reactor accident situations where large temperature gradients exist (Williams, 1986).

The thermophoretic effect can be explained in part by appealing to the kinetic theory of gases (Kennard, 1938). The higher energy molecules in the hot regions of the gas impinge on the particles with greater momenta than molecules coming from the cold regions, thus leading to the migration of the particles in the direction opposite to the temperature gradient. Theoretical analyses yield the following expression for the thermophoretic velocity of an isolated particle in a constant temperature gradient $\nabla T_{\infty}$ :

$$
\mathbf{U}^{(\mathbf{0})}=-K \frac{\eta}{\rho T_{\infty}(\mathbf{0})} \nabla T_{\infty}
$$

where the negative sign indicates that the motion is in the direction of decreasing temperature, $\eta$ is the fluid viscosity, $\rho$ is the fluid density, and $T_{\infty}(0)$ is the bulk gas absolute temperature at the particle center in the absence of the particle (or the mean gas temperature in the vicinity of the particle). The thermophoretic coefficient $K$ depends on the magnitude of the Knudsen number, $l / a$, where $l$ is the mean free path of the gas molecules and $a$ is the radius of the particle. Note 
that $\rho T_{\infty}$ is constant for an ideal gas at constant pressure.

In the free molecule regime $(l / a \gg 1)$, the velocity distribution of the incoming gas molecules may be taken to be uninfluenced by the small particle and given by the Maxwell or Chapman-Enskog distributions (Waldmann and Schmitt, 1966; Whitmore, 1981). Under this assumption, the thermophoretic coefficient was found to be

$$
K=\frac{3}{4(1+\pi \beta / 8)} .
$$

The theory adopts the usual assumption that a fraction $\beta$ of the gas molecules colliding with the particle is reflected diffusely (thermally) with a Maxwellian distribution and the remaining fraction $(1-\beta)$ is reflected specularly. The value of the coefficient of diffuse reflection $(\beta)$ is usually about 0.9 (Friedlander, 1977). Note that the thermophoretic velocity for the "small particle" regime is independent of particle size. Equation (1.2) has long been known to be in agreement with experiments.

In the "large particle" regime $(l / a \ll 1)$, the fluid flow may be described by a continuum model and the thermophoretic force arises from an induced "thermal creep" along the particle surface due to the existence of a tangential temperature gradient at the particle-fluid interface. Utilizing gas kinetic theory, Maxwell (1879) predicted that a tangential temperature gradient $\nabla_{s} T$ at a gas-solid surface would cause a thin layer of gas adjacent to the surface to move, with the relative velocity at the outer edge of the layer being

$$
\mathbf{v}^{(s)}=C_{s} \frac{\eta}{\rho T} \nabla_{s} T
$$

where $T$ is the local gas temperature. The thermal slip coefficient $C_{s}$ was found to be $\frac{3}{4}$ by Maxwell on the assumption that the distribution function in the bulk of the gas held all the way to the solid wall. The thermal creep velocity $\mathbf{v}^{(s)}$ is directed toward the high temperature side. By using the Maxwellian creep velocity equation (1.3), which gives the coupling between temperature and velocity fields, as a slip velocity boundary condition and solving the equation of continuum fluid motion (at low Reynolds number) incorporating with the heat conduction in the gas and particle, Epstein (1929) derived the following equation for the thermophoretic coefficient:

$$
K=2 C_{s} \frac{k}{2 k+\hat{k}}
$$

where $k$ and $\hat{k}$ are thermal conductivities of the gas and the particle, respectively. The thermophoretic velocity predicted by eq. (1.4), which is also independent of particle size, is in fair agreement with experimental data when the ratio $\hat{k} / k$ is not too high, less than about 10 (as for oil droplets). But it gives much too small thermophoretic velocities (by a factor 30 and more) for particles with large thermal conductivity, for instance, sodium chloride particles with $\hat{k} / k \approx 100$. In- deed, in the limit $\bar{k} / k \rightarrow \infty$, eq. (1.4) yields $\mathbf{U}^{(0)}=\mathbf{0}$, whereas Schadt and Cadle (1961) distinctly observed and measured a thermophoresis with sodium chloride particles.

The solution for thermophoretic velocity obtained by Epstein (1929) was improved by Brock (1962) using the low-Knudsen-number effects of temperature jump at the gas-particle surface as well as hydrodynamic (isothermal) gas slippage (in addition to the thermal creep velocity) along the particle surface. The resulting expression for the thermophoretic coefficient of a suspended aerosol sphere is

$$
K=\frac{2 C_{s}\left(k+\hat{k} C_{t} l / a\right)}{\left(1+2 C_{m} l / a\right)\left(2 k+\hat{k}+2 \hat{k} C_{t} l / a\right)}
$$

where the dimensionless coefficients $C_{t}$ and $C_{m}$ (numerical factors of order unity) account for the temperature jump and hydrodynamic slip, respectively, at the particle surface and must be determined experimentally for each gas-solid system. Both Epstein and Brock assumed the value of $C_{s}$ in eqs (1.4) and (1.5) to be equal to the Maxwell value $\left(C_{s}=\frac{3}{4}\right)$. Note that eq. (1.5) is applicable to the range of finite Knudsen number and reduces to eq. (1.4) when $l / a=0$. For large particles $(l / a \ll 1)$ and $\hat{k} / k \rightarrow \infty$, eq. (1.5) yields $K=2 C_{s} C_{t}(l / a)$, whereas Epstein formula (1.4) gives $K=0$. Satisfactory agreement of the prediction by eq. (1.5) with experiments (Schadt and Cadle, 1961) has been obtained. The best kinetic-theory values for complete thermal and momentum accommodations appear to be $C_{s}=1.17, C_{t}=2.18$ and $C_{m}=1.14$ (Talbot et al., 1980). The extension of Brock's (1962) analysis to a spheroidal particle has also been considered by using the prolate or oblate spheroidal coordinate system (Leong, 1984; Williams, 1986).

Derjaguin and Yalamov (1965) challenged the thermal creep model and solved the hydrodynamic problem of thermophoresis based on an application of irreversible thermodynamics and Onsager's reciprocity relations. By taking the temperature jump at the particle surface into account, their result for the thermophoretic coefficient is the same as eq. (1.5) with $C_{m}=0$ and $C_{s}=\frac{3}{2}$. Derjaguin et al. $(1966,1976)$ presented the experimental data of thermophoretic velocities for a variety of aerosols, which are in good agreement with eq. (1.5) with $C_{m}=0, C_{s}=\frac{3}{2}$ and suitable selection of coefficient $C_{t}$. These authors attributed some of the discrepancies in the experimental work of other investigators to the presence of convective fields or the occurrence of thermo-osmosis. These discrepancies may be responsible for the view held by some that the thermophoretic velocity determined in a "stagnant" gas is not directly applicable to a flow system, although it is certainly possible that for the slip flow regime, at least, the flow distorts the temperature distribution in the gas surrounding the particle and thereby alters thermophoretic velocity. According to eq. (1.5), particles with large thermal conductivity and small Knudsen number (say, $\hat{k} / k=100$ and $l / a=0.01$ ) will migrate by thermophoresis at velocities of $10-50 \mu \mathrm{m} \mathrm{s}^{-1}$ in temperature gradients of 
order $100 \mathrm{~K} \mathrm{~cm}^{-1}$; such gradients are easily attainable in thermal boundary layers.

In real situations of thermophoresis, collections of aerosol particles are usually encountered, and effects of particle interactions will be important. However, most theoretical treatments have typically addressed the migration of a single particle. Through an exact representation in spherical bipolar coordinates, the low-Knudsen-number thermophoresis of two spherical particles in response to a constant temperature gradient along the line of their centers was examined by Reed and Morrison (1975) and the present authors (Chen and Keh, 1995). Numerical results of correction to eq. (1.5) for each particle were presented for various cases. It was found that, for the axisymmetric thermophoresis of two identical spheres, each sphere migrates faster than its undisturbed velocity $\mathbf{U}^{(0)}$ given by eqs (1.1) and (1.5). For the case of two spheres with unequal radii, the particle interaction effect is stronger on the smaller particle than on the larger one. Thus, a stable distance will be developed between the spheres for the situation where the smaller sphere is ahead, and on the contrary, both spheres will finally collide (and may stick together) if the smaller sphere is following. In general, the particle interactions in thermophoresis are less significant than those for the corresponding motion driven by a body force.

Until now, the interactions between particles undergoing thermophoresis in asymmetric configurations have not been investigated. The object of the present work is to study the thermophoretic motion of two spherical particles in a constant temperature gradient oriented arbitrarily relative to the line of particle centers. The particles, which are freely suspended in the gaseous medium, may differ in size, in thermal conductivity and in surface properties. It is assumed that the Knudsen numbers are small. A method of reflections is used to solve the problem. In Section 2 we consider the local temperature and flow fields produced by a single spherical particle placed in a prescribed temperature field whose gradient is not necessarily constant over length scales comparable to the particle radius. We prove that eq. (1.1) with eq. (1.5) also applies to a single sphere in a non-uniform temperature gradient, provided that $\nabla T_{\infty}$ is evaluated at the position of the particle center. These results are then used in Section 3 to alternately evaluate the effects of one sphere on the other in a constant temperature gradient. The translational and angular velocities of the thermophoretic particles are determined in this manner with an error of $O\left(r_{12}^{-8}\right)$, where $r_{12}$ is the center-to-center distance between the particles, and the results are given in eq. (3.10). In Section 4 method-of-reflections results are compared with the numerical calculations of Chen and Keh (1995) for the case of two spheres oriented parallel with the undisturbed temperature gradient. Typical effects of the particle sizes and physical properties on the twosphere interactions in thermophoresis are discussed. Finally, in Section 5, the results of two-sphere interactions derived in Section 3 are applied to a theory of concentration effects on transport properties in dilute dispersions to obtain the effect of the volume fraction of particles of each type on the mean thermophoretic velocities in a bounded suspension, and the general result is given in eqs (5.9) and (5.10).

\section{A SINGLE AEROSOL SPHERE IN AN ARBITRARY TEMPERATURE FIELD}

On the purpose of obtaining the interactions between two thermophoretic spheres by the method of reflections, it is essential to realize the thermal and hydrodynamic effects caused by a single particle in an arbitrary temperature field $T_{A}(\mathbf{x})$. We consider a rigid sphere of radius a with internal heat conductivity $\hat{k}$ existing in a surrounding gas where the heat conductivity is $k$. All the physical properties of the particle and the fluid are taken to be constant, and the Knudsen number is assumed to be small. The instantaneous center of the particle is positioned at $x_{0}$, and the relative position vector is defined as $\mathbf{r}=\mathbf{x}-\mathbf{x}_{0}$. Although $\mathbf{x}_{0}$ changes with time, the problem can be dealt with as a quasi-steady state if both the Peclet and Reynolds numbers are small. It is assumed that $a\left|\nabla T_{A}\right| / T_{A}\left(\mathbf{x}_{0}\right) \ll 1$. Because the boundary conditions of the fluid velocity field are coupled with the temperature gradient at the particle surface, it is necessary to determine the temperature distribution first.

\subsection{Temperature field}

The energy equations governing the temperature distribution are

$$
\nabla^{2} T=0
$$

for the fluid and

$$
\nabla^{2} \hat{T}=0
$$

for the particle. It is obvious that $\nabla^{2} T_{A}=0$. The boundary conditions at the particle surface $(r=a)$ require that the normal heat fluxes be continuous and a temperature jump which is proportional to the normal temperature gradient (Kennard, 1938) occur. Also, the fluid temperature must approach the applied field far from the particle and the temperature inside the particle is finite everywhere. Thus, one has

$$
\begin{gathered}
k \frac{\partial T}{\partial r}=\hat{k} \frac{\partial \hat{T}}{\partial r} \quad \text { at } r=a \\
T-\hat{T}=C_{t} l \frac{\partial T}{\partial r} \quad \text { at } r=a \\
\hat{T} \text { is finite for } r<a \\
T \rightarrow T_{A} \quad \text { as } r \rightarrow \infty .
\end{gathered}
$$

In the Smoluchowski formula $(2.2 b), l$ is the mean free path of the surrounding fluid; $C_{t}$ is the dimensionless temperature jump coefficient which is semi-empirically related to the thermal accommodation coefficient $f_{t}$ at the particle-fluid interface by $C_{t} \simeq \frac{15}{8}\left(2-f_{t}\right) / f_{t}$ (Waldmann and Schmitt, 1966). 
A general solution to eq. (2.1) that satisfies eqs $(2.2 \mathrm{c}, \mathrm{d})$ is

$$
\begin{aligned}
& T=T_{A}+\sum_{m=1}^{\infty}\left(\frac{a}{r}\right)^{m+1} \mathbf{S}_{m}[\cdot] \lambda_{m} \\
& \hat{T}=T_{A}+\sum_{m=1}^{\infty}\left(\frac{r}{a}\right)^{m} \mathbf{S}_{m}[\cdot] \hat{\lambda}_{m} .
\end{aligned}
$$

Here, the $m$ th-order polyadic $S_{m}$ is a surface harmonic, defined by

$$
\mathbf{S}_{m}=r^{m+1} \nabla^{m}\left(r^{-1}\right)
$$

the first few ones are

$$
\begin{aligned}
& S_{0}=1 \\
& \mathbf{S}_{1}=-\frac{\mathbf{r}}{r} \\
& \mathbf{S}_{2}=3 \frac{\mathbf{r r}}{r^{2}}-\mathbf{l}
\end{aligned}
$$

where $I$ is the unit dyadic. $\lambda_{m}$ and $\hat{\lambda}_{m}$ are polyadic constants, and the symbol $[\cdot]$ represents $m$ scalar products using the inner nesting convention.

Substitution of eq. (2.3) into eqs $(2.2 \mathrm{a}, \mathrm{b})$ shows that

$$
\begin{aligned}
& \lambda_{1}=-G a\left(\nabla T_{A}\right)_{0} \\
& \lambda_{2}=\frac{1}{3} H a^{2}\left(\nabla \nabla T_{A}\right)_{0} \\
& \hat{\lambda}_{1}=-\hat{G} a\left(\nabla T_{A}\right)_{0} \\
& \hat{\lambda}_{2}=\frac{1}{3} \hat{H} a^{2}\left(\nabla \nabla T_{A}\right)_{0}
\end{aligned}
$$

where

$$
\begin{aligned}
& G=\frac{1-k^{*}+k^{*} C_{t}^{*}}{2+k^{*}+2 k^{*} C_{t}^{*}} \\
& H=\frac{1-k^{*}+2 k^{*} C_{t}^{*}}{3+2 k^{*}+6 k^{*} C_{t}^{*}} \\
& \hat{G}=\frac{1-k^{*}-2 k^{*} C_{t}^{*}}{2+k^{*}+2 k^{*} C_{t}^{*}} \\
& \hat{H}=\frac{1-k^{*}-3 k^{*} C_{t}^{*}}{3+2 k^{*}+6 k^{*} C_{t}^{*}}
\end{aligned}
$$

and the subscript 0 to variables inside parentheses denotes evaluation at $\mathbf{x}=\mathbf{x}_{0}$. In eqs (2.6), $C_{t}^{*}=C_{t} l / a$ and $k^{*}=\hat{k} / k$. Substituting eqs (2.5) into eqs (2.3) and realizing that $T_{A}$ satisfies Laplace's equation, we have

$$
\begin{aligned}
T= & T_{A}+G\left(\frac{a}{r}\right)^{3} \mathbf{r} \cdot\left(\nabla T_{A}\right)_{0} \\
& +H\left(\frac{a}{r}\right)^{5} \mathbf{r r}:\left(\nabla \nabla T_{A}\right)_{0}+O\left(\nabla \nabla \nabla T_{A}\right)_{0} \\
\hat{T}= & T_{A}+\hat{G} \mathbf{r} \cdot\left(\nabla T_{A}\right)_{0} \\
& +\hat{H} \mathbf{r r}:\left(\nabla \nabla T_{A}\right)_{0}+O\left(\nabla \nabla \nabla T_{A}\right)_{0} .
\end{aligned}
$$

The tangential component of the temperature gradient at the particle surface, $\nabla_{s} T(=(I-$ $\mathrm{nn}) \cdot(\nabla T)_{r=a}$, where $\mathbf{n}$ is the unit normal vector at the particle surface pointing into the fluid), is needed to evaluate the thermal creep velocity in a later derivation. It can be obtained by differentiating eq. (2.7a) at $r=a$, substituting $\nabla T_{A}$ by its Taylor expansion about $\mathbf{x}=\mathbf{x}_{0}$, and eliminating the normal component. The result is

$$
\begin{aligned}
\nabla_{s} T= & (1+G)(\mathbf{I}-\mathbf{n n}) \cdot\left(\nabla T_{A}\right)_{0} \\
& +(1+2 H) a(\mathbf{I}-\mathbf{n n}) \mathbf{n}:\left(\nabla \nabla T_{A}\right)_{0}+O\left(\nabla \nabla \nabla T_{A}\right)_{0} .
\end{aligned}
$$

\subsection{Velocity field}

Due to the low Reynolds number encountered in the thermophoresis, the fluid velocity is governed by the Stokes equations:

$$
\begin{aligned}
\eta \nabla^{2} \mathbf{v}-\nabla p & =\mathbf{0} \\
\nabla \cdot \mathbf{v} & =0
\end{aligned}
$$

where $\mathbf{v}(\mathbf{x})$ is the fluid velocity and $p(\mathbf{x})$ is the dynamic pressure. Owing to the thermal creep velocity given by eq. (1.3) and the hydrodynamic slip velocity along the particle surface as well as the fluid at rest far from the particle, the boundary conditions for the velocity field are:

$$
\begin{gathered}
\mathbf{v}=\mathbf{v}_{s}=\mathbf{U}+a \Omega \times \mathbf{n}+\frac{1}{\eta} C_{m} l(\mathbf{I}-\mathbf{n n}) \mathbf{n}: \mathbf{T} \\
+C_{s} \frac{\eta}{\rho\left(T_{A}\right)_{0}} \nabla_{s} T \text { at } r=a \\
\mathbf{v} \rightarrow \mathbf{0} \quad \text { as } r \rightarrow \infty .
\end{gathered}
$$

In eq. (2.10a), $\mathbf{T}\left(=\eta\left[(\nabla \mathbf{v})+(\nabla \mathbf{v})^{T}\right]\right)$ is the viscous stress tensor; $C_{m}$ is the dimensionless coefficient of the gas-kinetic isothermal slip which is semi-empirically related to the momentum accommodation coefficient $f_{m}$ at the particle-fluid interface by $C_{m} \simeq\left(2-f_{m}\right) / f_{m}$ (Kennard, 1938); $\mathbf{U}$ and $\mathbf{\Omega}$ are the instantaneous translational and angular velocities, respectively, of the particle to be determined. The temperature gradient along the particle surface, $\nabla_{s} T$, has been obtained in eq. (2.8). It is assumed that $\eta / \rho T$ is constant in spite of the variation of $T$ with position.

A solution for the velocity field can be constructed from Lamb's general solution as outlined by Brenner (1964). The fluid velocity is completely specified when the polyadic coefficients $\boldsymbol{a}_{m}, \boldsymbol{\beta}_{m}$ and $\gamma_{m}$ in the following formulas are calculated using the value of the velocity field on the particle surface $\left(\mathbf{v}_{\mathrm{s}}\right)$ :

$$
\begin{aligned}
\mathbf{n} \cdot \mathbf{v}_{s} & =\sum_{m=1}^{\infty} \boldsymbol{a}_{m}[\cdot] \mathbf{S}_{m} \\
-a \nabla \cdot \mathbf{v}_{s} & =\sum_{m=1}^{\infty} \boldsymbol{\beta}_{m}[\cdot] \mathbf{S}_{m}, \\
a \mathbf{n} \cdot\left(\nabla \times \mathbf{v}_{s}\right) & =\sum_{m=1}^{\infty} \gamma_{m}[\cdot] \mathbf{S}_{m} .
\end{aligned}
$$

The force and torque exerted by the fluid on the surface $r=a$ are given by

$$
\begin{gathered}
\mathbf{F}=2 \pi \eta a\left(3 a_{1}+\beta_{1}\right) \\
\mathbf{T}=4 \pi \eta a^{2} \gamma_{1} .
\end{gathered}
$$

Since the particle is freely suspended in the surrounding fluid, the velocities $U$ and $\Omega$ are obtained by 
setting the above expressions for $\mathbf{F}$ and $\mathbf{T}$ equal to zero.

After substituting eq. (2.10a) into the left-hand side of eq. (2.11c), one has

$$
2 a n \cdot \Omega=\sum_{m=1}^{\infty} \gamma_{m}[\cdot] \mathbf{S}_{m}
$$

or

$$
\begin{aligned}
& \gamma_{1}=-2 a \Omega \\
& \gamma_{m}=\mathbf{0} \text { for } m>1 .
\end{aligned}
$$

By setting $\mathbf{T}=\mathbf{0}$ and using eqs (2.12b) and (2.13b), we find

$$
\boldsymbol{\Omega}=\mathbf{0}
$$

as the general result for an imposed temperature field $T_{A}$ as long as $\nabla^{2} T_{A}=0$.

The translational motion is described by the coefficients $\boldsymbol{a}_{m}$ and $\boldsymbol{\beta}_{m}$. It can be obtained from eqs (2.10a) and (2.11a) that

$$
\begin{aligned}
& a_{1}=-\mathrm{U} \\
& \boldsymbol{a}_{\boldsymbol{m}}=\mathbf{0} \text { for } m>1 .
\end{aligned}
$$

Finally, substituting eq. (2.10a) together with eq. (2.8) into eq. (2.11b) and neglecting the terms of $O\left(\nabla \nabla \nabla T_{A}\right)$ (only $\boldsymbol{\beta}_{1}$ and $\boldsymbol{\beta}_{2}$ are needed), one has

$$
\begin{aligned}
\boldsymbol{\beta}_{1}= & \frac{1}{1+3 C_{m}^{*}}\left[3 C_{m}^{*} \mathbf{U}-6 C_{s} \frac{\eta}{\rho\left(T_{A}\right)_{0}}\right. \\
& \left.\times\left(\frac{1+k^{*} C_{t}^{*}}{2+k^{*}+2 k^{*} C_{t}^{*}}\right)\left(\nabla T_{A}\right)_{0}\right] \\
\boldsymbol{\beta}_{2}= & 2 B a\left(\nabla \nabla T_{A}\right)_{0}
\end{aligned}
$$

where

$$
B=\frac{5}{2} C_{s} \frac{\eta}{\rho\left(T_{A}\right)_{0}}\left[\frac{1+2 k^{*} C_{t}^{*}}{\left(1+5 C_{m}^{*}\right)\left(3+2 k^{*}+6 k^{*} C_{t}^{*}\right)}\right]
$$

and $C_{m}^{*}=C_{m} l / a$. The force-free characteristic of this problem is used with eqs (2.12a) and (2.15a) $\left(\beta_{1}=-3 a_{1}=3 \mathrm{U}\right)$, and the particle translational velocity is found to be

$$
\mathbf{U}=A\left(\nabla T_{A}\right)_{0}
$$

where

$$
A=-2 C_{s} \frac{\eta}{\rho\left(T_{A}\right)_{0}}\left[\frac{1+k^{*} C_{t}^{*}}{\left(1+2 C_{m}^{*}\right)\left(2+k^{*}+2 k^{*} C_{t}^{*}\right)}\right]
$$

Equation (2.18) shows that the particle velocity is proportional to the prescribed temperature gradient evaluated at the particle center and is identical with eq. (1.5) in the dependence on the physical properties of the surrounding fluid and the particle itself. To our knowledge, the result of eq. (2.18) has heretofore only been derived for constant $\nabla T_{A}$.
The corresponding velocity field about the thermophoretic particle is determined by

$\mathbf{v}=\nabla \phi_{-2}+\nabla \phi_{-3}+\frac{1}{2 \eta} \mathbf{r} p_{-3}+O\left(\nabla \nabla \nabla T_{A}\right)$

where the solid spherical harmonic functions $\phi_{-(m+1)}$ and $p_{-(m+1)}$ are (Happel and Brenner, 1983):

$$
\begin{aligned}
& \phi_{-2}=\frac{a^{3}}{4} r^{-2}\left(a_{1}+\boldsymbol{\beta}_{1}\right) \cdot\left(-\mathbf{e}_{r}\right) \\
& \phi_{-3}=\frac{a^{4}}{6} r^{-3} \boldsymbol{\beta}_{2}:\left[3 \mathbf{e}_{r} \mathbf{e}_{r}-I\right] \\
& p_{-3}=\eta a^{2} r^{-3} \boldsymbol{\beta}_{2}:\left[3 \mathbf{e}_{r} \mathbf{e}_{r}-I\right], \ldots, \text { etc. }
\end{aligned}
$$

After evaluating $\phi_{-2}, \phi_{-3}$, and $p_{-3}$ from $\boldsymbol{a}_{1}, \boldsymbol{\beta}_{1}$, and $\boldsymbol{\beta}_{2}$, we get

$$
\begin{aligned}
\mathbf{v}= & \frac{1}{2} A\left(\frac{a}{r}\right)^{3}\left[3 \frac{\mathbf{r r}}{r^{2}}-\mathbf{I}\right] \cdot\left(\nabla T_{A}\right)_{0} \\
& +B\left\{3\left(\frac{a}{r}\right)^{3} \frac{\mathbf{r r r}}{r^{2}}+\left(\frac{a}{r}\right)^{5}\left[2 \mathrm{lr}-5 \frac{\mathbf{r r r}}{r^{2}}\right]\right\}:\left(\nabla \nabla T_{A}\right)_{0} \\
& +O\left(\nabla \nabla \nabla T_{A}\right)_{0} .
\end{aligned}
$$

It should be noted that the leading order in $\mathbf{v}$ decays as $r^{-3}$ rather than as a Stokeslet or stresslet (force dipole). This is a characteristic of the "phoretic motion" which is the movement of particles caused by driving forces interacting with the surface of each particle.

For the motion of a freely suspended aerosol sphere under arbitrarily applied temperature gradient $\nabla T_{A}$ and velocity field $\mathbf{v}_{A}$ in an unbounded fluid, the translational and angular velocities of the particle can be obtained by combining eqs (2.14), (2.18) and the generalized Faxen laws for a force-free sphere with slip surface (Felderhof, 1977):

$$
\begin{aligned}
& \mathbf{U}=A\left(\nabla T_{A}\right)_{0}+\left(\mathbf{v}_{A}\right)_{0}+\frac{a^{2}}{6\left(1+2 C_{m}^{*}\right)}\left(\nabla^{2} \mathbf{v}_{A}\right)_{0} \\
& \mathbf{\Omega}=\frac{1}{2}\left(\nabla \times \mathbf{v}_{A}\right)_{0} .
\end{aligned}
$$

The superposition of the thermal and hydrodynamic contributions in the above formulas is valid due to the linearity of the problem.

\section{SOLUTION FOR INTERACTIONS BETWEEN TWO THERMOPHORETIC SPHERES}

We now consider the quasi-steady, low-Knudsennumber thermophoresis of two spherical particles of radii $a_{1}$ and $a_{2}$. They are oriented at an arbitrary angle to the prescribed temperature gradient $\mathbf{E}_{\infty}\left[=\nabla T_{\infty}\right.$, where $T_{\infty}(\mathbf{x})$ is the undisturbed temperature field of the surrounding gas]. The particles, which may be made from different materials, are supposed to be sufficiently close to interact thermally and hydrodynamically with each other, but sufficiently distant from boundary walls for the surrounding fluid 
to be regarded as unbounded. Let $\mathbf{e}$ be the unit vector pointing from the center of particle 1 to the center of particle 2 and $r_{12}$ be the center-to-center distance between the particles. $\mathbf{E}_{\infty}$ is assumed to be constant over distances comparable to $r_{12}$ and the fluid at infinity is at rest. The effects of gravity and Brownian motion are ignored in this section, but the latter will be considered in the evaluation of the average thermophoretic velocity of an aerosol suspension in Section 5 .

In the situation $\left(a_{1}+a_{2}\right) / r_{12} \ll 1$, a method of reflections (Anderson, 1985; Chen and Keh, 1988) is used to solve the two-sphere problem. Because of the linear characteristic of governing equations (2.1) and (2.9) as well as boundary conditions (2.2) and (2.10), the solution of the fluid temperature and velocity fields for eqs (2.1a) and (2.9) can be decomposed into a sum of fields, which depend on increasing powers of $r_{12}^{-1}$ :

$$
\begin{gathered}
T=T_{1}^{(1)}+T_{2}^{(2)}+T_{1}^{(3)}+T_{2}^{(4)}+\cdots \\
\mathbf{v}=\mathbf{v}_{1}^{(1)}+\mathbf{v}_{2}^{(2)}+\mathbf{v}_{1}^{(3)}+\mathbf{v}_{2}^{(4)}+\cdots
\end{gathered}
$$

where subscripts 1 and 2 represent the reflections from particle 1 and particle 2, respectively, and the superscript $(i)$ denotes the $i$ th reflection from either particle surface. Hence, the particles' translational and angular velocities can also be expressed in the form of a series:

$$
\begin{aligned}
& \mathbf{U}_{1}=\mathbf{U}_{1}^{(0)}+\mathbf{U}_{1}^{(2)}+\mathbf{U}_{1}^{(4)}+\cdots \\
& \boldsymbol{\Omega}_{1}=\boldsymbol{\Omega}_{1}^{(0)}+\boldsymbol{\Omega}_{1}^{(2)}+\boldsymbol{\Omega}_{1}^{(4)}+\cdots \\
& \mathbf{U}_{2}=\mathbf{U}_{2}^{(1)}+\mathbf{U}_{2}^{(3)}+\mathbf{U}_{2}^{(5)}+\cdots \\
& \boldsymbol{\Omega}_{2}=\boldsymbol{\Omega}_{2}^{(1)}+\boldsymbol{\Omega}_{2}^{(3)}+\boldsymbol{\Omega}_{2}^{(5)}+\cdots
\end{aligned}
$$

where $\mathbf{U}_{1}^{(i)}$ and $\boldsymbol{\Omega}_{1}^{(i)}$ are related to $T_{2}^{(i)}$ and $\mathbf{v}_{2}^{(i)}$ by eqs (2.23) for $i=2,4,6, \ldots$, while $\mathbf{U}_{2}^{(i)}$ and $\boldsymbol{\Omega}_{2}^{(i)}$ are related to $T_{1}^{(i)}$ and $\mathbf{v}_{1}^{(i)}$ for $i=1,3,5, \ldots$ Obviously, the unperturbed linear temperature field gives

$$
\begin{aligned}
& \mathbf{U}_{1}^{(0)}=A_{1} \mathbf{E}_{\infty} \\
& \mathbf{\Omega}_{1}^{(0)}=\mathbf{0} .
\end{aligned}
$$

Hereinafter, we use $A_{j}, B_{j}$ and $G_{j}$ to represent the values of $A, B$ and $G$, respectively, defined by eqs (2.19), (2.17) and (2.6a) for the particle $j(j=1$ or 2$)$.

The initial temperature gradient $\nabla T_{1}^{(1)}$ and velocity field $v_{1}^{(1)}$, which correspond to the thermophoresis of particle 1 isolated in an unbounded fluid under the prescribed field $\nabla T_{\infty}$ are easily obtained from eqs (2.7a) and (2.22) for $r_{1}>a_{1}$ as:

$$
\begin{aligned}
\nabla T_{1}^{(1)} & =\mathbf{E}_{\infty}-G_{1}\left(\frac{a_{1}}{r_{1}}\right)^{3}\left[3 \frac{\mathbf{r}_{1} \mathbf{r}_{1}}{r_{1}^{2}}-\mathbf{I}\right] \cdot \mathbf{E}_{\infty} \\
\mathbf{v}_{1}^{(1)} & =\frac{1}{2} A_{1}\left(\frac{a_{1}}{r_{1}}\right)^{3}\left[3 \frac{\mathbf{r}_{1} \mathbf{r}_{1}}{r_{1}^{2}}-\mathbf{I}\right] \cdot \mathbf{E}_{\infty}
\end{aligned}
$$

where $\mathbf{r}_{1}$ is the position vector relative to the center of particle 1 and $r_{1}=\left|\mathbf{r}_{1}\right|$. Note that $\mathbf{v}_{1}^{(1)}$ is irrotational and satisfies Laplace's equation. The contributions of $\nabla T_{1}^{(1)}$ and $v_{1}^{(1)}$ to the velocity of particle 2 are deter- mined from eqs (2.23). It is found that

$$
\begin{gathered}
{\left[\nabla T_{1}^{(1)}\right]_{\mathrm{r}_{1}=r_{12} \mathrm{e}}=\mathbf{E}_{\infty}-G_{1}\left(\frac{a_{1}}{r_{12}}\right)^{3}[3 \mathrm{ee}-\mathbf{I}] \cdot \mathbf{E}_{\infty}} \\
{\left[\mathbf{v}_{1}^{(1)}\right]_{\mathrm{r}_{1}=r_{12} \mathrm{e}}=\frac{1}{2} A_{1}\left(\frac{a_{1}}{r_{12}}\right)^{3}[3 \mathrm{ee}-\mathrm{I}] \cdot \mathbf{E}_{\infty}}
\end{gathered}
$$

where $\mathbf{r}_{1}=r_{12}$ e represents the position of the center of particle 2. Thus,

$$
\mathbf{U}_{2}^{(1)}=A_{2} \mathbf{E}_{\infty}+\left(\frac{1}{2} A_{1}-A_{2} G_{1}\right)\left(\frac{a_{1}}{r_{12}}\right)^{3}[3 \mathrm{ee}-\mathrm{I}] \cdot \mathbf{E}_{\infty}
$$

$\mathbf{\Omega}_{2}^{(1)}=\mathbf{0}$.

Equation (3.7a) indicates that the effect of particle interaction in thermophoresis is $O\left(r_{12}^{-3}\right)$.

The first reflected temperature gradient field from particle $2, \nabla T_{2}^{(2)}$, can be derived from using eqs (2.7a) and (3.5a), while the first reflected velocity field from particle $2, \mathbf{v}_{2}^{(2)}$, can be evaluated from eqs (2.22) and (3.5). The results are

$$
\begin{aligned}
\nabla T_{2}^{(2)}= & -G_{2}\left(\frac{a_{2}}{r_{2}}\right)^{3}\left[3 \frac{\mathbf{r}_{2} \mathbf{r}_{2}}{r_{2}^{2}}-\mathbf{I}\right] \cdot\left[\nabla T_{1}^{(1)}\right]_{r_{1}=r_{12} \mathbf{e}} \\
& +O\left[r_{2}^{-4} \nabla \nabla T_{1}^{(1)}+r_{2}^{-3} \nabla \nabla \nabla T_{1}^{(1)}\right] \\
\mathbf{v}_{2}^{(2)}= & \frac{1}{2} A_{2}\left(\frac{a_{2}}{r_{2}}\right)^{3}\left[3 \frac{\mathbf{r}_{2} \mathbf{r}_{2}}{r_{2}^{2}}-\mathbf{I}\right] \cdot\left[\nabla T_{1}^{(1)}\right]_{r_{1}=r_{12} \mathbf{e}} \\
& +3 B_{2}\left(\frac{a_{2}}{r_{2}}\right)^{3} \frac{\mathbf{r}_{2} \mathbf{r}_{2} \mathbf{r}_{2}}{r_{2}^{2}}:\left[\nabla \nabla T_{1}^{(1)}\right]_{r_{1}=r_{12} \mathbf{e}} \\
& -\frac{5\left(1+2 C_{m 2}^{*}\right)}{2\left(1+5 C_{m 2}^{*}\right)}\left(\frac{a_{2}}{r_{2}}\right)^{3} \frac{\mathbf{r}_{2} \mathbf{r}_{2} \mathbf{r}_{2}}{r_{2}^{2}}:\left[\nabla \mathbf{v}_{1}^{(1)}\right]_{r_{1}=r_{12} \mathbf{e}} \\
& +O\left[r_{2}^{-4}\left(\nabla \nabla T_{1}^{(1)}+\nabla \mathbf{v}_{1}^{(1)}\right)\right. \\
& \left.+r_{2}^{-3}\left(\nabla \nabla \nabla T_{1}^{(1)}+\nabla \nabla \mathbf{v}_{1}^{(1)}\right)\right]
\end{aligned}
$$

Substituting eqs (3.8) into eqs (2.23) with $T_{A}=T_{2}^{(2)}$ and $\mathbf{v}_{A}=\mathbf{v}_{2}^{(2)}$, one obtains the contribution to the velocity of particle 1 due to the reflected fields from particle 2,

$$
\begin{aligned}
\mathbf{U}_{1}^{(2)}= & \left(\frac{1}{2} A_{2}-A_{1} G_{2}\right)\left(\frac{a_{2}}{r_{12}}\right)^{3}[3 \mathrm{ee}-\mathrm{I}] \cdot \mathbf{E}_{\infty} \\
& +\left\{\left(A_{1} G_{1} G_{2}-\frac{1}{2} A_{2} G_{1}\right)[3 \mathrm{ee}+\mathrm{I}]\right. \\
& \left.-\left[18 B_{2} G_{1}+\frac{15\left(1+2 C_{m 2}^{*}\right)}{2\left(1+5 C_{m 2}^{*}\right)} A_{1}\right] \mathbf{e e}\right\} \frac{a_{1}^{3} a_{2}^{3}}{r_{12}^{6}} \cdot \mathbf{E}_{\infty} \\
& +O\left(r_{12}^{-8}\right) \\
\mathbf{\Omega}_{1}^{(2)}= & -\left[9 B_{2} G_{1}+\frac{15\left(1+2 C_{m 2}^{*}\right)}{4\left(1+5 C_{m 2}^{*}\right)} A_{1}\right] \frac{a_{1}^{3} a_{2}^{3}}{r_{12}^{7}} \mathbf{e} \times \mathbf{E}_{\infty} \\
& +O\left(r_{12}^{-9}\right) .
\end{aligned}
$$

The $O\left(r_{12}^{-8}\right)$ and $O\left(r_{12}^{-9}\right)$ interactions in eqs (3.9) could be obtained by more detailed calculations of $\nabla T_{2}^{(2)}$ and $v_{2}^{(2)}$ and their derivatives at the position $r_{2}=-r_{12}$ (the center of particle 1 ), but the numer- 
ical significance would be small unless the gap between the particles approaches zero.

Obviously, $\mathbf{U}_{1}^{(4)}$ and $\boldsymbol{\Omega}_{1}^{(4)}$ will be of the orders $O\left(r_{12}^{-9}\right)$ and $O\left(r_{12}^{-10}\right)$, respectively. With the addition of eqs (3.4) and (3.9), the translational and angular velocities of particle 1 can be expressed as:

$$
\begin{aligned}
\mathbf{U}_{1}= & A_{1} \mathbf{E}_{\infty}+\left(\frac{1}{2} A_{2}-A_{1} G_{2}\right)\left(\frac{a_{2}}{r_{12}}\right)^{3}[3 \mathrm{ee}-\mathrm{I}] \cdot \mathbf{E}_{\infty} \\
& +\left\{\left(A_{1} G_{1} G_{2}-\frac{1}{2} A_{2} G_{1}\right)[3 \mathrm{ee}+\mathrm{I}]\right. \\
& \left.-\left[18 B_{2} G_{1}+\frac{15\left(1+2 C_{m 2}^{*}\right)}{2\left(1+5 C_{m 2}^{*}\right)} A_{1}\right] \mathbf{e e}\right\} \frac{a_{1}^{3} a_{2}^{3}}{r_{12}^{6}} \cdot \mathbf{E}_{\infty} \\
& +O\left(r_{12}^{-8}\right) \\
\mathbf{\Omega}_{1}= & -\left[9 B_{2} G_{1}+\frac{15\left(1+2 C_{m 2}^{*}\right)}{4\left(1+5 C_{m 2}^{*}\right)} A_{1}\right] \frac{a_{1}^{3} a_{2}^{3}}{r_{12}^{7}} \mathbf{e} \times \mathbf{E}_{\infty} \\
& +O\left(r_{12}^{-9}\right) .
\end{aligned}
$$

$\mathbf{U}_{2}$ and $\boldsymbol{\Omega}_{2}$, the velocities of particle 2 , can be obtained from the above equations by interchanging the subscripts 1 and 2 in all variables and replacing $e$ by - e. As expected, both particles will move with the velocity that would exist in the absence of the other (without rotation) for any arbitrary orientation of the particles as $r_{12} \rightarrow \infty$. It should be noted from eq. (3.10a) that the direction of thermophoresis of each sphere is deflected by the other, unless the temperature gradient is prescribed either parallel or perpendicular to the line of centers of the particles.

The result of eq. (3.10a) can be expressed in terms of dimensionless mobility tensors $\mathbf{M}_{i j}$ defined as follows:

$$
\mathbf{U}_{i}=\sum_{j=1}^{2} \mathbf{M}_{i j} \cdot \mathbf{U}_{j}^{(0)}, \quad i=1 \text { or } 2
$$

where $U_{j}^{(0)}$ is computed from eq. (3.4a). A comparison between eqs (3.10a) and (3.11) gives

$$
\mathbf{M}_{i j}=M_{i j}^{(p)} \mathbf{e e}+M_{i j}^{(n)}[\mathbf{I}-\mathbf{e e}]
$$

with

$$
\begin{aligned}
M_{11}^{(p)}=1 & -2 G_{2}\left(\frac{a_{2}}{r_{12}}\right)^{3} \\
& +\left[4 G_{1} G_{2}-\frac{15\left(1+2 C_{m 2}^{*}\right)}{2\left(1+5 C_{m 2}^{*}\right)}\right] \frac{a_{1}^{3} a_{2}^{3}}{r_{12}^{6}}+O\left(r_{12}^{-8}\right)
\end{aligned}
$$$$
M_{12}^{(p)}=\left(\frac{a_{2}}{r_{12}}\right)^{3}-\left(2 G_{1}+18 \frac{B_{2}}{A_{2}} G_{1}\right) \frac{a_{1}^{3} a_{2}^{3}}{r_{12}^{6}}+O\left(r_{12}^{-8}\right)
$$

$$
M_{11}^{(n)}=1+G_{2}\left(\frac{a_{2}}{r_{12}}\right)^{3}+G_{1} G_{2} \frac{a_{1}^{3} a_{2}^{3}}{r_{12}^{6}}+O\left(r_{12}^{-8}\right)
$$

$$
M_{12}^{(n)}=-\frac{1}{2}\left(\frac{a_{2}}{r_{12}}\right)^{3}-\frac{1}{2} G_{1} \frac{a_{1}^{3} a_{2}^{3}}{r_{12}^{6}}+O\left(r_{12}^{-8}\right) .
$$

The corresponding coefficients $M_{22}^{(p)}, M_{21}^{(p)}, M_{22}^{(n)}$ and $M_{21}^{(n)}$ can easily be derived from the above formulas by changing the subscripts. Note that, the particle-interaction coefficients $M_{i j}^{(p)}$ and $M_{i j}^{(n)}$ are independent of the thermal slip coefficient $C_{s}$.

\section{DISCUSSION ON INTERACTIONS BETWEEN TWO SPHERES}

The interaction between two aerosol spheres in a temperature gradient field, given by eqs (3.10), results from three phenomena. First, each particle disturbs the local temperature field experienced by the other. Second, the movement of each particle drags surrounding fluid (hydrodynamic slip at the particle surface is allowed though) that convects and rotates the other. Third, the thermal creep effect occurred at the surface of each particle causes a reverse tangential fluid velocity that affects the motion of the other. The leading term of the interaction for particle translation is $O\left(r_{12}^{-3}\right)$, because both the temperature gradient and velocity disturbances in the fluid phase produced by an isolated thermophoretic sphere decay like $r^{-3}$, as shown in eqs (2.7a) and (2.22). For thermophoretic spheres that allow free rotation, the leading term of the angular velocity is of $O\left(r_{12}^{-7}\right)$. Thus, the interaction between particles undergoing thermophoresis is much weaker than that between sedimenting particles, since the leading terms of particle interaction for the translational and angular velocities of two slip spheres driven by body forces are of $O\left(r_{12}^{-1}\right)$ and $O\left(r_{12}^{-2}\right)$, respectively.

The exact (numerical) solution of the mobility parameters $M_{11}^{(p)}, M_{12}^{(p)}, M_{21}^{(p)}$ and $M_{22}^{(p)}$ was obtained by solving the problem of axisymmetric thermophoretic motion of two spheres using bipolar coordinates (Chen and Keh, 1995). Table 1 gives a comparison of our asymptotic results from the method of reflections with this exact solution. For simplicity, only the case of two identical spheres $\left(a_{1}=a_{2}=a, k_{1}^{*}=k_{2}^{*}=k^{*}\right.$, $C_{s 1}=C_{s 2}=C_{s}, C_{t 1}^{*}=C_{t 2}^{*}=C_{t}^{*}, C_{m 1}^{*}=C_{m 2}^{*}=C_{m}^{*}$, $\left.U_{1}^{(0)}=U_{2}^{(0)}=U^{(0)}=\left|U^{(0)}\right|\right\rangle$ is presented. In this specific case, the particles will migrate at the same velocity $\left(U_{1}=U_{2}=U\right)$ because $M_{12}^{(p, n)}=M_{21}^{(p, n)}$, $M_{11}^{(p, n)}=M_{22}^{(p, n)}$, and $U_{1}^{(0)}=U_{2}^{(0)}$. It is found in Table 1 that the predictions of $U / U^{(0)}\left(=M_{11}^{(p)}+M_{12}^{(p)}\right)$ from the asymptotic approximation for various values of $k^{*}, C_{t}^{*}$ and $C_{m}^{*}$ are in perfect agreement with those of the exact solution. The errors in velocities are less than $0.1 \%$ for cases $2 a / r_{12} \leqslant 0.6$ or $1.2 \%$ for cases $2 a / r_{12} \leqslant 0.8$, indicating that the higher terms such as $O\left(r_{12}^{-8}\right)$ in eqs (3.10) or eqs (3.13) are not important unless the particles are nearly touching. Note that the value of $M_{11}^{(p)}+M_{12}^{(p)}$ evaluated from eqs (3.13a) and (3.13b) always underestimates the interaction effect between the particles. For the case of two spheres differing in size and/or in physical properties, eqs (3.13a) and (3.13b) can also be found to agree well with the exact solution.

The translational and rotational velocities for various cases of two spherical particles evaluated from eqs (3.10) are shown in Figs 1-3. In the cases of Figs 1 and 3 , the velocity of a particle normalized by the value 
Table 1. Normalized thermophoretic velocities $U / U^{(0)}$ of two identical spheres with their line of centres aligned with applied temperature gradient

\begin{tabular}{|c|c|c|c|c|c|}
\hline \multirow[b]{2}{*}{$k^{*}$} & \multirow[b]{2}{*}{$\frac{2 a}{r_{12}}$} & $C_{t} l / a=0.2$ & $C_{m} l / a=0.1$ & $C_{t} l / a=0.02$ & $C_{m} l / a=0.01$ \\
\hline & & $\begin{array}{l}\text { Asymptotic } \\
\text { solution }\end{array}$ & $\begin{array}{l}\text { Exact } \\
\text { solution }\end{array}$ & $\begin{array}{l}\text { Asymptotic } \\
\text { solution }\end{array}$ & $\begin{array}{c}\text { Exact } \\
\text { solution }\end{array}$ \\
\hline 1 & $\begin{array}{l}0.2 \\
0.4 \\
0.6 \\
0.8 \\
0.9\end{array}$ & $\begin{array}{l}1.0009 \\
1.0067 \\
1.0199 \\
1.0342 \\
1.0353\end{array}$ & $\begin{array}{l}1.0009 \\
1.0067 \\
1.0200 \\
1.0372 \\
1.0452\end{array}$ & $\begin{array}{l}1.0010 \\
1.0074 \\
1.0214 \\
1.0336 \\
1.0300\end{array}$ & $\begin{array}{l}1.0010 \\
1.0074 \\
1.0216 \\
1.0392 \\
1.0471\end{array}$ \\
\hline 10 & $\begin{array}{l}0.2 \\
0.4 \\
0.6 \\
0.8 \\
0.9\end{array}$ & $\begin{array}{l}1.0019 \\
1.0143 \\
1.0431 \\
1.0776 \\
1.0848\end{array}$ & $\begin{array}{l}1.0019 \\
1.0143 \\
1.0432 \\
1.0830 \\
1.1028\end{array}$ & $\begin{array}{l}1.0024 \\
1.0185 \\
1.0558 \\
1.1011 \\
1.1115\end{array}$ & $\begin{array}{l}1.0024 \\
1.0185 \\
1.0565 \\
1.1140 \\
1.1499\end{array}$ \\
\hline 100 & $\begin{array}{l}0.2 \\
0.4 \\
0.6 \\
0.8 \\
0.9\end{array}$ & $\begin{array}{l}1.0021 \\
1.0161 \\
1.0481 \\
1.0851 \\
1.0908\end{array}$ & $\begin{array}{l}1.0021 \\
1.0161 \\
1.0482 \\
1.0908 \\
1.1102\end{array}$ & $\begin{array}{l}1.0028 \\
1.0214 \\
1.0629 \\
1.1052 \\
1.1039\end{array}$ & $\begin{array}{l}1.0028 \\
1.0214 \\
1.0633 \\
1.1173 \\
1.1419\end{array}$ \\
\hline 1000 & $\begin{array}{l}0.2 \\
0.4 \\
0.6 \\
0.8 \\
0.9\end{array}$ & $\begin{array}{l}1.0021 \\
1.0163 \\
1.0486 \\
1.0857 \\
1.0910\end{array}$ & $\begin{array}{l}1.0021 \\
1.0163 \\
1.0488 \\
1.0915 \\
1.1105\end{array}$ & $\begin{array}{l}1.0029 \\
1.0216 \\
1.0620 \\
1.0960 \\
1.0834\end{array}$ & $\begin{array}{l}1.0029 \\
1.0216 \\
1.0621 \\
1.1059 \\
1.1153\end{array}$ \\
\hline
\end{tabular}

that prevails in the absence of the other is plotted vs the separation parameter $\left(a_{1}+a_{2}\right) / r_{12}$. It can be seen that the effect of particle interactions on the normalized thermophoretic velocities in general is increased with the increase in $\left(a_{1}+a_{2}\right) / r_{12}$.

For the situation of two identical spheres oriented along the prescribed temperature gradient, the interaction effect makes each particle move faster than its undisturbed value, as illustrated in Figs 1(a) and 2(a). On the other hand, the normalized migration velocity of two identical spheres undergoing thermophoresis normal to the line of their centers is a monotonic decreasing function of the separation parameter $2 a / r_{12}$ and the conductivity ratio $k^{*}$, as illustrated in Figs 1(b) and 2(b). A careful examination of the surrounding fluid recirculation pattern generated by the thermophoretic motion of a single spherical particle (Chen and Keh, 1995) shows why the particle interactions cause the enhancement or retardation in the particle velocity. In Figs 1(c) and 2(c), the normalized angular velocities of two identical spheres $\left(\Omega_{1}=-\Omega_{2}=\Omega\right.$ ) undergoing thermophoresis normal to the line of their centers are plotted, respectively, vs $2 a / r_{12}$ with $k^{*}$ as a parameter and vs $k^{*}$ with $C_{t}^{*}$ and $C_{m}^{*}$ as parameters. For two spheres aligned with the prescribed temperature gradient, the flow is axisymmetric and the angular velocities of particles vanish. Figures 1 and 2 indicate that the particle interaction effect in general is more significant if the value of $k^{*}$ becomes greater or the values of $C_{t}^{*}$ and $C_{m}^{*}$ become smaller (with some exceptions). In the limit $k^{*}=0$ or in the limit $C_{i}^{*} \rightarrow \infty$, eqs (2.6a), (2.17) and (2.19) give $G=\frac{1}{2}$ and $B / A=-5\left(1+2 C_{m}^{*}\right) / 6\left(1+5 C_{m}^{*}\right)$. Under this situation, the thermophoretic velocity of each of these two identical spheres (which can be arbitrarily oriented) is unaffected by the presence of the other, as predicted by eq. (3.10); this phenomenon is similar to that observed for the thermocapillary motion of two identical gas bubbles (Meyyappan and Subramanian, 1984; Anderson, 1985).

In Fig. 3, the normalized translational and rotational velocities (of particle 1) for the thermophoresis of two unequal-sized spheres with the same physical properties $\left(k^{*}, C_{s}, C_{t}\right.$ and $\left.C_{m}\right)$ are plotted vs $\left(a_{1}+a_{2}\right) / r_{12}$ with the ratio $a_{2} / a_{1}$ as a parameter. Again, this figure indicates that the migration velocity of the first sphere in general is enhanced if the second sphere is oriented along the prescribed temperature gradient (with some exceptions when $a_{2} / a_{1}$ is small) and is reduced if the second one is oriented perpendicular to the gradient. It can be seen that the effect of particle interactions on the normalized thermophoretic mobility is far greater on the smaller particle than on the larger one. For example, when the large sphere has a radius five times that of the smaller one, the migration velocity of the smaller sphere (with $\left.\left(a_{1}+a_{2}\right) / r_{12}=0.8\right)$ can be increased by as much as $71 \%$ (if the temperature gradient is prescribed parallel to the line of particle centers) or decreased by $36 \%$ (if the gradient is imposed perpendicular to the line of particle centers), while the migration velocity of the large sphere can hardly be influenced even when the two particles are nearly touching. Interestingly, as shown in Fig. 3(c), a maximum of the angular velocity of particle 1 (to the accuracy of $O\left(r_{12}^{-7}\right)$ ) exists for the case of $a_{2} / a_{1}=\frac{3}{4}$ when the parameter $\left(a_{1}+a_{2}\right) / r_{12}$ is 


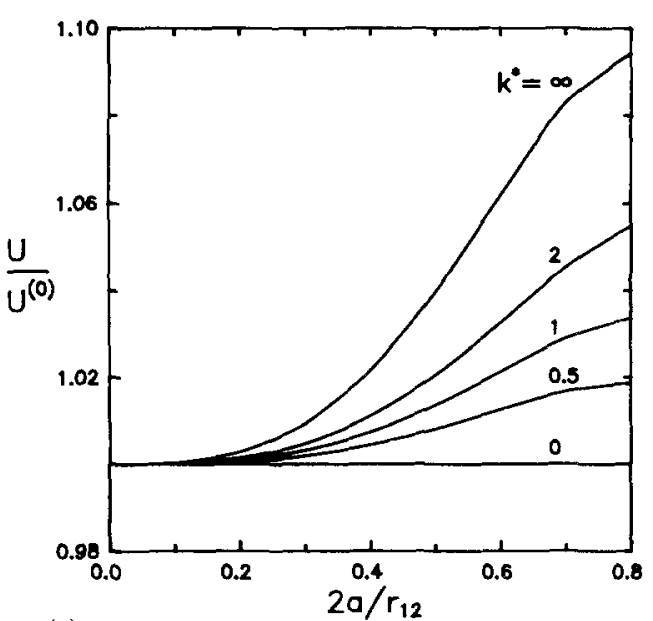

(a)

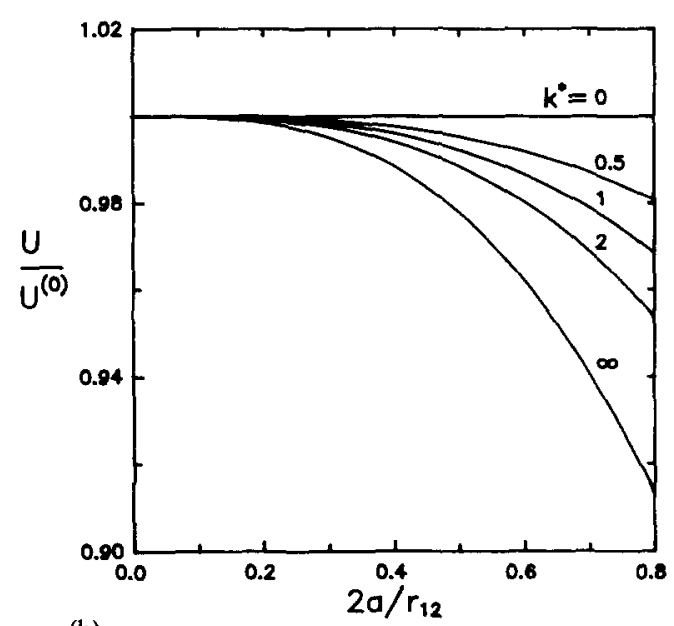

(b)

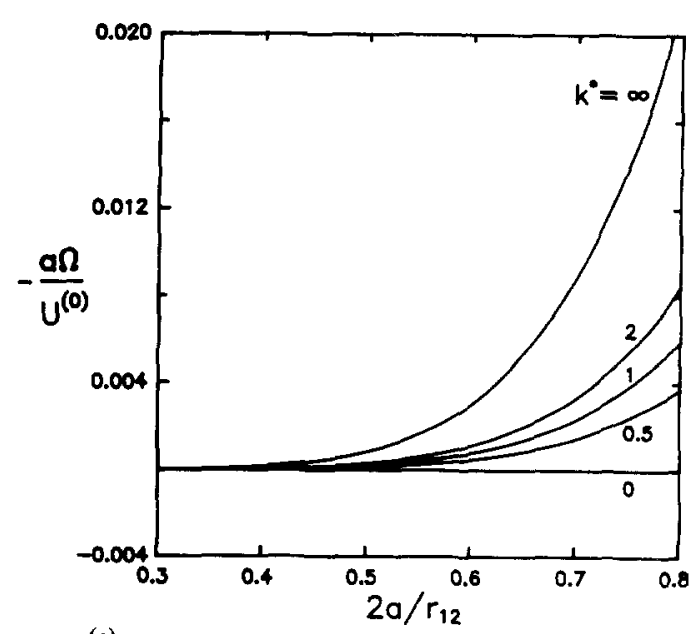

(c)

Fig. 1. Normalized translational and rotational velocities of two identical spheres vs the separation parameter $2 a / r_{12}$ with $k^{*}$ as a parameter $\left(C_{t}^{*}=0.02, C_{m}^{*}=0.01\right)$ : (a) temperature gradient prescribed parallel to the line of particle centers; (b) and (c) temperature gradient prescribed perpendicular to the line of particle centers.
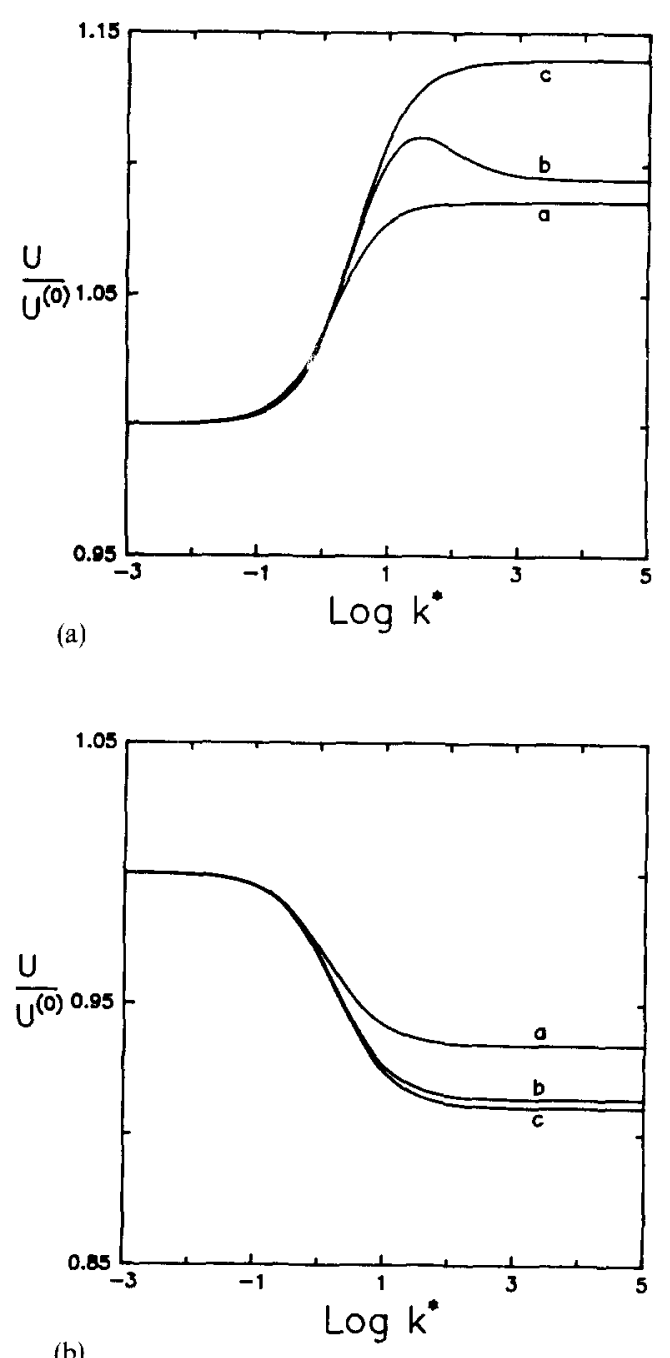

(b)

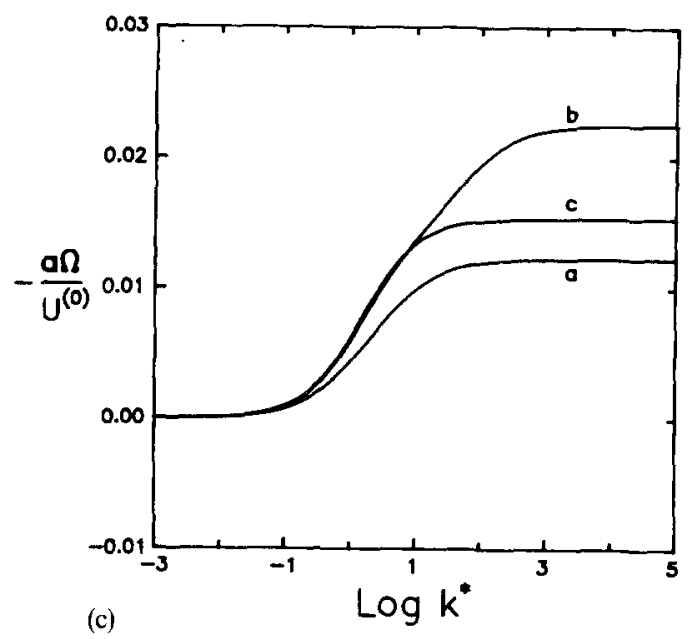

Fig. 2. Normalized translational and rotational velocities of two identical spheres vs the conductivity ratio $k^{*}$ with $C_{t}^{*}$ and $C_{m}^{*}$ as parameters $\left(2 a / r_{12}=0.8\right)$ : (a) temperature gradient prescribed parallel to the line of particle centers; (b) and (c) temperature gradient prescribed perpendicular to the line of particle centers. Curve $a: C_{t}^{*}=0.2$ and $C_{m}^{*}=0.1$; curve $b: C_{t}^{*}=0.02$ and $C_{m}^{*}=0.01$; curve $c: C_{t}^{*}=C_{m}^{*}=0$. 


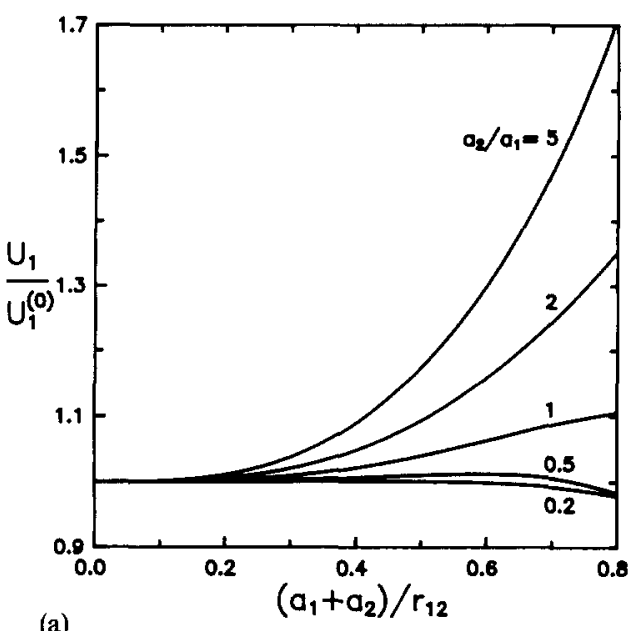

(a)

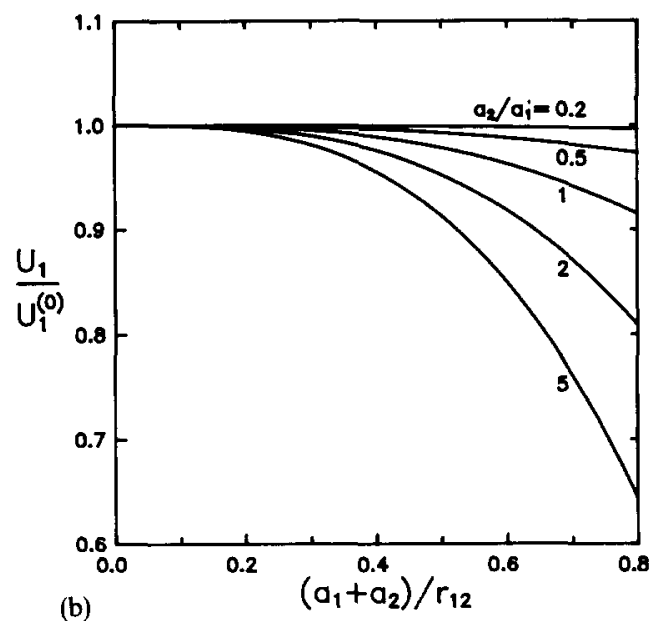

(b)

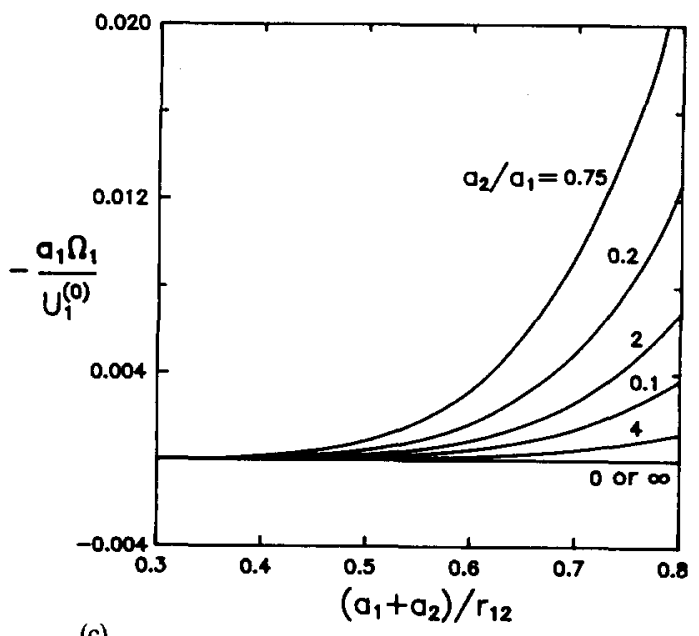

(c)

Fig. 3. Normalized translational and rotational velocities of particle 1 for the system of two spheres of identical thermal conductivities and surface properties $\left(k^{*}=100, C_{t} l / a_{1}\right.$ $\left.=0.02, \quad C_{m} l / a_{1}=0.01\right)$ vs the separation parameter $\left(a_{1}+a_{2}\right) / r_{12}$ with $a_{2} / a_{1}$ as a parameter: (a) temperature gradient prescribed parallel to the line of particle centers; (b) and (c) temperature gradient prescribed perpendicular to the line of particle centers. kept constant. This behavior, which can also be demonstrated by using eq. (3.10b), is understandable knowing that the value of $r_{12} / a_{1}$ increases linearly with $a_{2} / a_{1}$ for a fixed $\left(a_{1}+a_{2}\right) / r_{12}$.

In practical applications of thermophoresis, collections of aerosol particles are usually encountered. It is therefore necessary to determine the dependence of the thermophoretic mobility on particle concentration. The interaction effects between pairs of particles, obtained in the previous section, can be extended to the evaluation of the mean thermophoretic velocity in a suspension of aerosol particles. In the following section, formulas for this mean velocity correct to the order of first power of the volume fraction of the particles will be presented.

\section{CONCENTRATION DEPENDENCE OF THERMOPHORETIC VELOCITY}

For a bounded suspension of aerosol spheres, it is no longer possible to define the particle velocity relative to the distant fluid, as the particles are spread through the entire space and there is no distant fluid. Instead, the particle velocity should be calculated for a reference frame in which the net particle and fluid flux is zero and $\mathbf{E}_{\infty}$ is the volume average of the temperature gradient field over the entire suspension. Thus,

$$
\frac{1}{V} \int_{V} \mathbf{v}(\mathbf{x}) \mathrm{d} \mathbf{x}=\mathbf{0}
$$

and

$$
\frac{1}{V} \int_{V} \nabla T(\mathbf{x}) \mathrm{d} \mathbf{x}=\mathbf{E}_{\infty}
$$

where $V$ denotes the entire volume of the suspension. Due to the existence of particles, gaseous medium, and a temperature jump at the surface of each particle, eq. (5.1b) can be further expressed as

$$
\begin{aligned}
\mathbf{E}_{\infty}= & \frac{1}{V}\left\{\int_{V_{s}} \nabla T(\mathbf{x}) \mathrm{d} \mathbf{x}+\sum_{j}\left[\int_{V_{j}} \nabla T_{j}(\mathbf{x}) \mathrm{d} \mathbf{x}\right.\right. \\
& \left.\left.+\int_{S_{j}}\left(T(\mathbf{x})-T_{j}(\mathbf{x})\right) \mathbf{n}_{j} \mathrm{~d} S\right]\right\}
\end{aligned}
$$

where $V_{j}$ and $S_{j}$ are the volume and surface area of particle $j$, respectively; $V_{s}=V-\sum_{j} V_{j}$ is the volume occupied by the continuous medium, $\mathbf{n}_{j}$ is the unit normal vector at the surface of particle $j$ pointing into the fluid. The last integral in eq. (5.2) accounts for the temperature gradient on the surface of particle $j$, which is singular.

Based on a microscopic model of particle interactions in a dilute dispersion which involves both statistical and low Reynolds number hydrodynamic concepts (Batchelor, 1972; Reed and Anderson, 1980), the mean thermophoretic velocity of a "test" particle (subscript $t$ ), which samples all positions in the suspen- 
sion, is given by

$$
\begin{aligned}
\left\langle\mathbf{U}_{t}\right\rangle= & \mathbf{U}_{t}^{(0)}+C\left\{\int_{V} \mathbf{v}^{*}(\mathbf{r})[g(\mathbf{r})-1] \mathrm{d} \mathbf{r}\right. \\
& +\frac{a_{t}^{2}}{6\left(1+2 C_{m t}^{*}\right)} \int_{V} \nabla^{2} \mathbf{v}^{*}(\mathbf{r}) g(\mathbf{r}) \mathrm{d} \mathbf{r} \\
& +A_{t} \int_{V}\left[\mathbf{E}^{*}(\mathbf{r})-\mathbf{E}_{\infty}\right][g(\mathbf{r})-1] \mathrm{d} \mathbf{r} \\
& \left.+\int_{V} \mathbf{W}(\mathbf{r}) g(\mathbf{r}) \mathrm{d} \mathbf{r}\right\}+O\left(C^{2}\right) .
\end{aligned}
$$

Here, $\mathbf{U}_{t}^{(0)}=A_{t} \mathbf{E}_{\infty}$, which is the undisturbed thermophoretic velocity of the test particle, $g(\mathbf{r})$ is the radial distribution function describing the two-particle configurational probability, and $C$ is the macroscopic concentration of the neighboring particles (assumed to have identical radius and physical properties and to have no particle agglomeration). $\mathbf{E}^{*}$ (r) and $\mathbf{v}^{*}(\mathbf{r})$ are the temperature gradient and velocity fields, respectively, at position $r$ when a single neighboring sphere at the origin 0 moves due to the prescribed temperature gradient $\mathbf{E}_{\infty}$, which are expressed by eq. (3.5) (eliminating the superscripts and subscripts) for $r>a$. Inside the neighboring sphere $(r<a), \mathbf{E}^{*}$ and $\mathbf{v}^{*}$ can be obtained using eqs $(2.7 \mathrm{~b})$ and (2.18).

$$
\begin{aligned}
\mathbf{E}^{*} & =(1+\hat{G}) \mathbf{E}_{\infty} \\
\mathbf{v}^{*} & =A \mathbf{E}_{\infty}
\end{aligned}
$$

both are constant. Note that $\mathbf{E}^{*}$ is singular with a temperature jump at $r=a$. W(r) is a correction function needed to account for the perturbation on $\mathbf{v}^{*}$ owing to the presence of the test particle, and is given by

$$
\begin{aligned}
\mathbf{W}(\mathbf{r})= & \mathbf{U}_{t}^{*}(\mathbf{r})-\mathbf{U}_{t}^{(0)}-\mathbf{v}^{*}(\mathbf{r}) \\
& -\frac{a_{t}^{2}}{6\left(1+2 C_{m t}^{*}\right)} \nabla^{2} \mathbf{v}^{*}(\mathbf{r})-A_{t}\left[\mathbf{E}^{*}(\mathbf{r})-\mathbf{E}_{\infty}\right]
\end{aligned}
$$

where $\mathbf{U}_{t}^{*}(\mathbf{r})$ is the actual velocity of the test particle located at $\mathbf{r}$ with respect to the origin of a single neighbor at $0 . U_{t}^{*}(\mathbf{r})$ can be calculated from eq. (3.11), taking subscripts 1 and 2 to denote the test and neighboring particles, respectively. Note that the term of modified Faxen's correction involving $\nabla^{2} \mathbf{v}^{*}$, which has appeared in eqs (5.3) and (5.5), equals zero as computed from using eq. (3.5b).

To evaluate the volume integrals in eq. (5.3), we assume that the radial distribution function has the following equilibrium value for rigid spheres without long-range pair potential:

$$
\begin{array}{ll}
g=0 & \text { if } r<a_{t}+a \\
g=1+O(C) & \text { if } r>a_{t}+a
\end{array}
$$

where $O(C)$ is a term proportional to the concentration of neighbors. In other words, the particles must be sufficiently small so that Brownian motion dominates any multiparticle hydrodynamic interactions that might impart microscopic structure to the suspension. In general, it is necessary to obtain the pair distribu- tion function as the solution of a conservation equation of Fokker-Planck type for a polydisperse system of spheres (Batchelor, 1982). The conditions under which the assumption of local equilibrium is valid for a dilute dispersion consisting of different types of particles are also discussed by Reed and Anderson (1980).

Given eqs (3.5) or eqs (5.4) for $\mathbf{E}^{*}$ and $\mathbf{v}^{*}$, eq. (3.11) for $\mathbf{U}_{t}^{*}$, eq. (5.5) for $\mathbf{W}$ and eqs (5.6) for $g$, the integrals in eq. (5.3) are evaluated incorporating eq. (5.2) to obtain

$$
\left\langle\mathbf{U}_{t}\right\rangle=\mathbf{U}_{t}^{(0)}\left[1+\alpha_{t} \varphi+O\left(\varphi^{2}\right)\right]
$$

with

$$
\begin{aligned}
\alpha_{t}=-\frac{A}{A_{t}}-G+ & {\left[2 G_{t} G-G_{t} \frac{A}{A_{t}}-6 G_{t} \frac{B}{A_{t}}\right.} \\
& \left.-\frac{5\left(1+2 C_{m}^{*}\right)}{2\left(1+5 C_{m}^{*}\right)}\right]\left(\frac{a_{t}}{a_{t}+a}\right)^{3}
\end{aligned}
$$

where $\varphi=4 \pi a^{3} C / 3$ is the volume fraction of the neighbor particles. The three terms in the expression (5.8) for $\alpha_{t}$ are obtained in order of the contributions from the first, third and fourth integrals in eq. (5.3). This result is not exact, even given that eqs (5.6) hold, because $O\left(r^{-8}\right)$ terms are neglected in $\mathrm{U}_{t}^{*}$; however, the error should be small and will appear only in the calculation involving the correction function $\mathbf{W}$. In the derivation of eq. (5.8), all the neighboring particles were assumed to be identical, even though they are allowed to differ from the test particle.

For a dispersion of particles that have a distribution in radius and physical properties, a generalization of eqs (5.7) and (5.8) leads to

$$
\left\langle\mathbf{U}_{i}\right\rangle=\mathbf{U}_{i}^{(0)}\left[1+\sum_{j} \alpha_{i j} \varphi_{j}+O\left(\varphi^{2}\right)\right]
$$

where

$$
\begin{aligned}
\alpha_{i j}= & -\frac{A_{j}}{A_{i}}-G_{j}+\left[2 G_{i} G_{j}-G_{i} \frac{A_{j}}{A_{i}}-6 G_{i} \frac{B_{j}}{A_{i}}\right. \\
& \left.-\frac{5\left(1+2 C_{m j}^{*}\right)}{2\left(1+5 C_{m j}^{*}\right)}\right]\left(\frac{a_{i}}{a_{i}+a_{j}}\right)^{3} .
\end{aligned}
$$

Here the subscript $i$ denotes the type of particles having radius $a_{i}$ and physical properties $k_{i}^{*}, C_{s i}$, $C_{t i}$ and $C_{m i}$. Equation (5.10) predicts that $\alpha_{i j}$ can be positive or negative depending on the sizes and physical properties of particles of types $i$ and $j$.

In a suspension of identical particles, the expression for the mean thermophoretic velocity can be reduced from eqs (5.9) and (5.10) to

$$
\begin{array}{r}
\langle\mathbf{U}\rangle=\mathbf{U}^{(0)}\left[1+\alpha \varphi+O\left(\varphi^{2}\right)\right] \\
\alpha=-1-G+\frac{1}{8}\left[2 G^{2}-\left(1+6 \frac{B}{A}\right) G-\frac{5\left(1+2 C_{m}^{*}\right)}{2\left(1+5 C_{m}^{*}\right)}\right] .
\end{array}
$$

For the limiting situation of $k^{*}=0$ or $C_{t}^{*} \rightarrow \infty$, we have $\alpha=-\frac{3}{2}$ and the contribution from the correction function $W$ to $\alpha$ vanishes. Note that $\alpha$ is non-zero in 
this limit, even though there is no interaction between two particles in an unbounded fluid, as discussed in the previous section. The reason for this is that in the bounded suspension the volume-averaged flow is zero (this contributes -1 to $\alpha$ ) and the volume-averaged temperature gradient is $\mathbf{E}_{\infty}$ (this contributes $-\frac{1}{2}$ to $\alpha$ ), as required by eqs (5.1). For the case of large particles $(l / a \ll 1)$ with $k^{*} \rightarrow \infty$, eqs (2.6a), (2.17) and (2.19) give $G=-1$ and $B / A=-\frac{5}{4}$; then eq. (5.12) yields $\alpha=-\frac{7}{8}$. On the other hand, for the case of $l / a=0$ and $k^{*} \gg 1$, one has $G=-1$ and $B / A=-\frac{5}{8}$; then eq. (5.12) gives $\alpha=-\frac{13}{32}$ [and eq. (1.5) yields $K=2 C_{s} / k^{*}$ ]. In both of these two limiting cases, $G=-1$ and the contribution to $\alpha$ is entirely due to the correction function W. Results of $\alpha$ calculated from eq. (5.12) for a suspension of particles at various values of $C_{t}^{*}$ and $C_{m}^{*}$ are plotted vs $k^{*}$ in Fig. 4. It can be found in each case that the mean thermophoretic velocity is reduced with an increase in the particle concentration (with $-\frac{3}{2} \leqslant \alpha \leqslant-\frac{13}{32}$ ). Although the magnitude of $\alpha$ in general decreases with the increase of $k^{*}$, the dependence is not necessarily monotonic. Note that the effect of interactions between two particles (in an unbounded fluid) is to reduce the magnitude of $\alpha$. Also, the magnitude of $\alpha$ in thermophoresis is always small relative to what is found for sedimentation of spherical particles (Reed and Anderson, 1980), where the value of $\alpha$ ranges from -4.5 (for the case $C_{m}^{*} \rightarrow \infty$ ) to -6.5 (for the case $C_{m}^{*}=0$ ). The larger magnitude of $\alpha$ for sedimentation is due to the $O\left(r_{12}^{-1}\right)$ hydrodynamic interactions, which are much stronger than the $O\left(r_{12}^{-3}\right)$ interactions for thermophoresis.

For regular practical aerosol systems with low Knudsen numbers, it is reasonable to have $2 a / R<0.6$, where $R$ is the mean inter-particle distance. This condition leads to $\varphi<0.12$. Thus, the results of our analysis neglecting the $O\left(r_{12}^{-8}\right)$ and $O\left(\varphi^{2}\right)$ effects should be quite accurate and useful in real applications.

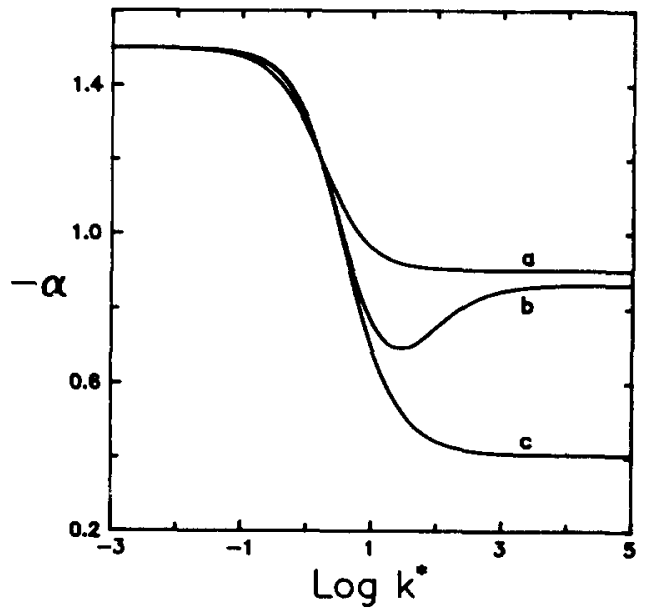

Fig. 4. Coefficient $\alpha$ calculated from eq. (5.12) for a suspension of identical spheres vs $k^{*}$. Curve $a: C_{t}^{*}=0.2$ and $C_{m}^{*}=0.1$; curve $b: C_{t}^{*}=0.02$ and $C_{m}^{*}=0.01$; curve $c$ : $C_{t}^{*}=C_{m}^{*}=0$.

Acknowledgement-This research was supported by the National Science Council of the Republic of China under Grant NSC84-2214-E002-006.

\section{$a$}

$A, B$

\section{C}

$C_{m}$

$C_{m}^{*}$

$C_{s}$

$C_{t}$

$C_{t}^{*}$

e

$\mathbf{e}_{\mathbf{r}}$

$\mathbf{E}_{\infty}$

$f_{m}$

$f_{t}$

$\mathbf{F}$

$\mathbf{T}$

$g$

$G, \hat{G}$, $H, \hat{H}$

I

$k$

$\hat{k}$

$k^{*}$

$K$

$l$

$\mathbf{M}_{i j}$

$M_{i j}^{(n, p)}$

n

$p$

$p_{-n}$

r

$r$

$\mathbf{r}_{12}$

$r_{12}$

$S$

$\mathbf{S}_{\boldsymbol{m}}$

$T$
$\hat{T}$

\section{NOTATION}

particle radius, $m$

coefficients defined by eqs (2.17) and (2.19), $\mathrm{m}^{2} \mathrm{~s}^{-1} \mathrm{~K}^{-1}$

number density of particles in suspension, $\mathrm{m}^{-3}$

dimensionless coefficient accounting for the hydrodynamic slip

equal to $C_{m} l / a$

dimensionless coefficient accounting for the thermal slip

dimensionless coefficient accounting for the temperature jump

equal to $C_{t} l / a$

unit vector pointing from particle 1 to particle 2

equal to $r / r$

uniform applied temperature gradient, $\mathrm{K} \mathrm{m}^{-1}$

momentum accommodation coefficient thermal accommodation coefficient

force exerted on particle by the fluid, $\mathrm{N}$ torque exerted on particle by the fluid, $\mathrm{N} \mathrm{m}$

two-particle radial distribution function coefficients defined by eq. (2.6)

unit dyadic

thermal conductivity of the fluid, $\mathrm{W} \mathrm{m}{ }^{-1} \mathrm{~K}^{-1}$

thermal conductivity of the particle, $\mathrm{W} \mathrm{m}^{-1} \mathrm{~K}^{-1}$

equal to $\hat{k} / k$

thermophoretic coefficient defined by eq. (1.1)

mean free path of the gas molecules, $m$ dimensionless mobility tensors defined by eq. (3.11)

dimensionless mobility coefficients defined by eq. (3.12)

unit normal vector at particle surface pointing into the fluid

dynamic pressure, $\mathrm{N} \mathrm{m}^{-2}$

solid spherical harmonics, $\mathrm{kg} \mathrm{m}^{-1} \mathrm{~s}^{-2}$

position vector relative to sphere center, $\mathrm{m}$

equal to $|\mathbf{r}|$, $\mathrm{m}$

vector pointing from particle 1 to particle $2, \mathrm{~m}$

center-to-center distance between particle 1 and particle $2, \mathrm{~m}$

surface area of the particle, $\mathrm{m}^{2}$

mth-order polyadic surface harmonics defined by eq. (2.4)

temperature distribution in the fluid, $\mathrm{K}$ temperature distribution inside the particle, $\mathrm{K}$ 
$T_{\infty} \quad$ uniform applied temperature field, $\mathrm{K}$

$\mathrm{U}$ thermophoretic velocity, $\mathrm{m} \mathrm{s}^{-1}$

$\langle U\rangle \quad$ ensemble-averaged thermophoretic velocity, $\mathrm{m} \mathrm{s}^{-1}$

v fluid velocity field, $\mathrm{m} \mathrm{s}^{-1}$

$v^{(s)} \quad$ thermal slip velocity defined by eq. (1.3), $\mathrm{m} \mathrm{s}^{-1}$

$\mathbf{v}_{s} \quad$ fluid velocity at particle surface, $\mathrm{m} \mathrm{s}^{-1}$

$V \quad$ volume of the dispersion system, $\mathrm{m}^{3}$

W correction function defined by eq. (5.5), $\mathrm{m} \mathrm{s}^{-1}$

$\mathbf{x}$ position vector, $\mathrm{m}$

Greek letters

$\alpha \quad$ interaction coefficient for $O(\varphi)$

$a_{m}, \beta_{m}, \gamma_{m}$ polyadic coefficients defined by eq. (2.11), $\mathrm{m} \mathrm{s}^{-1}$

$\eta \quad$ fluid viscosity, $\mathrm{kg} \mathrm{m}^{-1} \mathrm{~s}^{-1}$

$\lambda_{m}, \hat{\lambda}_{m} \quad$ polyadic coefficients defined by eq. (2.3), K

$\rho \quad$ fluid density, $\mathrm{kg} \mathrm{m}^{-3}$

$\mathrm{T}$ viscous stress tensor of the fluid, $\mathrm{N} \mathrm{m}^{-2}$

$\phi_{-n} \quad$ solid spherical harmonics, $\mathrm{m}^{2} \mathrm{~s}^{-1}$

$\varphi \quad$ volume fraction of particles in suspension

$\boldsymbol{\Omega}$ angular velocity of particle, $\mathrm{s}^{-1}$

Subscripts

$\begin{array}{ll}0 & \text { particle center } \\ 1 & \text { particle } 1 \\ 2 & \text { particle } 2 \\ A & \text { arbitrary applied field } \\ i & i \text {-type particle } \\ j & j \text {-type particle } \\ t & \text { test particle }\end{array}$

\section{Superscripts}

(0)

(i)

infinite dilution

ith reflection

unbounded fluid

\section{REFERENCES}

Anderson, J. L., 1985, Droplet interactions in thermocapillary motion. Int. J. Multiphase Flow 11, 813-824.

Bakanov, S. P., 1991, Thermophoresis in gases at small Knudsen numbers. Aerosol. Sci. Technol. 15, 77-92.

Batchelor, G. K., 1972, Sedimentation in a dilute dispersion of spheres. J. Fluid Mech. 52, 245-268.

Batchelor, G. K., 1982, Sedimentation in a dilute polydisperse system of interacting spheres. Part 1. General theory. J. Fluid Mech. 119, 379-408.

Batchelor, G. K. and Shen, C., 1985, Thermophoretic deposition of particles in gas flowing over cold surfaces. $J$. Colloid Interface Sci. 107, 21-37.

Brenner, H., 1964, The Stokes resistance of a slightly deformed sphere. Chem. Engng Sci. 19, 519-539.

Brock, J. R., 1962, On the theory of thermal forces acting on aerosol particles. J. Colloid Sci. 17, 768-780.

Chen, S. B. and Keh, H. J., 1988, Electrophoresis in a dilute dispersion of colloidal spheres. A.I.Ch.E. J. 34, 1075-1085.

Chen, S. H. and Keh, H. J., 1995, Axisymmetric ther- mophoretic motion of two spheres. J. Aerosol Sci. 26, 429-444.

Derjaguin, B. V., Rabinovich, Ya. I., Storozhilova, A. I. and Shcherbina, G. 1., 1976, Measurement of the coefficient of thermal slip of gases and the thermophoresis velocity of large-size aerosol particles. J. Colloid Interface Sci. 57, 451-461.

Derjaguin, B. V., Storozhilova, A. I. and Rabinovich, Ya. I., 1966, Experimental verification of the theory of thermophoresis of aerosol particles. J. Colloid Interface Sci. 21, 35-58.

Derjaguin, B. V. and Yalamov, Yu., 1965, Theory of thermophoresis of large aerosol particles. J. Colloid Sci. 20 , 555-570.

Epstein, P. S., 1929, Zur Theorie des Radiometers. Z. Phys. 54, 537-563.

Felderhof, B. U., 1977, Hydrodynamic interaction between two spheres. Physica A 88, 373-384.

Friedlander, S. K., 1977, Smoke, Dust and Haze. Wiley, New York.

Happel, J. and Brenner, H., 1983, Low Reynolds Number Hydrodynamics. Martinus Nijhoff, Dordrecht.

Kennard, E. H., 1938, Kinetic Theory of Gases. McGrawHill, New York.

Leong, K. H., 1984, Thermophoresis and diffusiophoresis of large aerosol particles of different shapes. J. Aerosol Sci. $15,511-517$.

Maxwell, J. C., 1879, On stresses in rarified gases arising from inequalities of temperature. Phil. Trans. R. Soc. 170, 231-256.

Meyyappan, M. and Subramanian, R. S., 1984, The thermocapillary motion of two bubbles oriented arbitrarily relative to a thermal gradient. J. Colloid Interface Sci. 97, 291-294.

Montassier, N., Boulaud, D. and Renoux, A., 1991, Experimental study of thermophoretic particle deposition in laminar tube flow. J. Aerosol Sci. 22, 677-687.

Reed, C. C. and Anderson, J. L., 1980, Hindered settling of a suspension at low Reynolds number. A.I.Ch.E. J, 26, 816-827.

Reed, L. D. and Morrison, F. A., Jr, 1975, Particle interactions in low Knudsen number thermophoresis. J. Aerosol Sci. 6, 349-365.

Sasse, A. G. B. M., Nazaroff, W. W. and Gadgil, A. J., 1994, Particle filter based on thermophoretic deposition from natural convection flow. Aerosol Sci. Technol. 20, 227-238.

Schadt, C. F. and Cadle, R. D., 1961, Thermal forces on aerosol particles. J. phys. Chem. 65, 1689-1694.

Simpkins, P. G., Greenberg-Kosinski, S. and MacChesney, J. B., 1979, Thermophoresis: The mass transfer mechanism in modified chemical vapor deposition. J. Appl. Phys. 50, $5676-5681$.

Talbot, L., Cheng, R. K., Schefer, R. W. and Willis, D. R., 1980 , Thermophoresis of particles in a heated boundary layer. J. Fluid Mech. 101, 737-758.

Waldmann, L. and Schmitt, K. H., 1966, Thermophoresis and diffusiophoresis of aerosols, in Aerosol Science (Edited by C. N. Davies). Academic Press, New York.

Weinberg, M. C., 1982, Thermophoretic efficiency in modified chemical vapor deposition process. J. Amer. Ceram. Soc., 65, 81-87.

Whitmore, P. J., 1981, Thermo- and diffusiophoresis for small aerosol particles. J. Aerosol Sci. 12, 1-9.

Williams, M. M. R., 1986, Thermophoretic forces acting on a spheroid. J. Phys. D 19, 1631-1642.

Ye, Y., Pui, D. Y. H., Liu, B. Y. H., Opiolka, S., Blumhorst, S. and Fissan, H., 1991, Thermophoretic effect of particle deposition on a free standing semiconductor wafer in a clean room. J. Aerosol Sci. 22, 63-72. 